\title{
THE EFFECT OF SLIPS ON THE ANISOTROPIC BEHAVIOUR OF POLYCRYSTALLINE ALUMINIUM AT ELEVATED TEMPERATURE
}

\author{
PAWEL H. DLUZEWSKI \\ Institute of Fundamental Technological Research, Swietokrzyska 21, 00-049 \\ Warsaw, Poland
}

(26 March 1991)

\begin{abstract}
A transition from the field of micro-heterogeneous strains through the crystal strain orientation distribution function to the averaging scheme used in slip theory is presented. The conjugate measures of stress and strain have been derived for slip theory.

The theory is utilized here to the modelling of the strongly anisotropic, thermally activated, flow process observed for untextured aluminium in the plastic strain range from $1 * 10^{-6}$ to $0.5 \%$. The subsequent yield surfaces are obtained by numerical simulation in time, the whole process corresponding to the definition of yielding used by Phillips and Tang (1972). It is shown that the observed athermal stress is closely related to residual stresses and depends strongly on the plastic strain offset applied. In the present considerations a role of orientation distribution of mobile dislocation densities in the anisotropic flow of polycrystalline metals is discussed.
\end{abstract}

KEY WORDS Orientation spaces, contravariant angular coordinates, Euler angles, slip theory, yield surface, athermal stress, dislocation density.

\section{INTRODUCTION}

Recently, many constitutive models based on the concept of orientation distributions of crystals and slips are developed to describe the plastic anisotropy of polycrystals. In spite of that, the majority of known experimental results for combined loading path have never been quantitatively modeled by any micromechanical theory taking into account time of experiment, temperature changes and combined preloading path. A typical instance of this are the results of Phillips and Tang (1972) concerning the dependence of the small offset yield surfaces on the initial strain and temperature, cf. Ikegami (1982).

The idea of describing the plastic flow of metals as an effect of thermally activated dislocation glides was proposed by Seeger (1954). Next, this concept has been developed in many more complicated models, e.g. Seeger (1955), Weertman (1957), Conrad (1961), Kocks, Argon and Ashby (1975), among many others. On the other hand, verifications of the stress-strain behaviour of the models were made mainly in relation to the axial loading tests. At the same time a modelling of the anisotropic behaviour of polycrystal was developed on the base of the balance equations for slips, see e.g. Lin and Ito (1966), Tokuda, Kratochvil and Ohno (1985). Using such models it was shown that yield surfaces corresponded approximately to envelopes of elementary yield conditions for microslips, see e.g. Kiryk and Dluzewski (1989). 
In the next section the dependence of the back stress on the residual stress, temperature and mobile dislocation density is discussed. Coming back to the concept of thermal activation process a simple interpretation of the dependence is suggested. In the third section a constant curvature Riemannian space of crystal orientations is used to obtain some averaging schemes for strain tensor field on the polycrystalline aggregate region. Section four is devoted to slip theory in which the constitutive relations are postulated between the conjugate strain and stress averages derived in the third section. In the framework of the theory a model to predict the rate-dependent stress-strain behaviour of aluminium is proposed. A verification of the model is made on the base of the experimental results obtained by Phillips and Tang (1972).

\section{EFFECT OF TEMPERATURE ON THE FLOW STRESS}

An approximately linear dependence of the flow stress on temperature is observed for many metals. In many cases to predict the flow process of crystals or polycrystals the models of thermally activated flow have been proposed. Our attention is focused on the relatively simple model, in which the plastic strain rate $\dot{\gamma}$ induced by shear stress $\tau$ is described by

$$
\dot{\gamma}=\rho_{m} b v_{0} \exp \frac{-U}{k T}\left\{\exp \frac{+v\left(\tau-\tau_{\mu}\right)}{k T}-\exp \frac{-v\left(\tau-\tau_{\mu}\right)}{k T}\right\},
$$

where $\rho_{m}$ is the mobile dislocation density, $b$ the Burgers vector, $v_{0}$ the dislocation velocity coefficient, $\tau_{\mu}$ denotes residual stress around mobile dislocations, cf. Figure 1. In many cases, with respect to weak dependence on temperature the stress $\tau_{\mu}$ is identified with athermal stress due to the interactions of mobile dislocations with athermal obstacles. $U$ denotes the activation energy, while $v$ is called the activation volume and $k$ is the Boltzmann constant, see e.g. Conrad (1961), Kroner and Teodosiu (1974). In our paper particular attention is given to the effect of dislocation density on the flow stress.

Let $\tau_{\mathrm{pl}}^{+}$and $\tau_{\mathrm{pl}}^{-}$denote the yield stresses for two mutually opposite directions of simple shearing. The yield stress $\tau_{\mathrm{pl}}^{-}$is often called the back stress. According to (1) the yield stresses are determined by

$$
\tau_{\mathrm{pl}}^{ \pm}=\tau_{\mu} \pm\left[\frac{1}{2} \Delta \tau_{\mu}\left(\Delta \gamma_{\mathrm{pl}}\right)+\frac{U-k T \ln \left(\rho_{m} b v_{0} / \dot{\gamma}_{\mathrm{pl}}\right)}{v}\right]
$$

where $\dot{\gamma}_{\mathrm{pl}}$ denotes the plastic strain rate resolved at the moment of reaching the yield stress, $\Delta \tau_{\mu}\left(\Delta \gamma_{\mathrm{pl}}\right)$ denotes the change of the residual stress induced by the strain offset. For the method using a very small inelastic strain offset e.g. $5 \times 10^{-6}$ the Bauschinger effect is detected before full unloading of the specimen, compare Figure 1a. with Figure 2.

Let $\Delta \tau_{\mathrm{pl}}$ denote the difference between $\tau_{\mathrm{pl}}^{+}$and $\tau_{\mathrm{pl}}^{-}$; then

$$
\Delta \tau_{\mathrm{pl}}=2 \frac{U-k T \ln \left(\rho_{m} b v_{0} / \dot{\gamma}_{\mathrm{pl}}\right)}{v}+\Delta \tau_{\mu}\left(\Delta \gamma_{\mathrm{pl}}\right)
$$

The dependence of $\Delta \tau_{\mathrm{pl}}$ on temperature is illustrated in Figure $2 \mathrm{~b}$. 


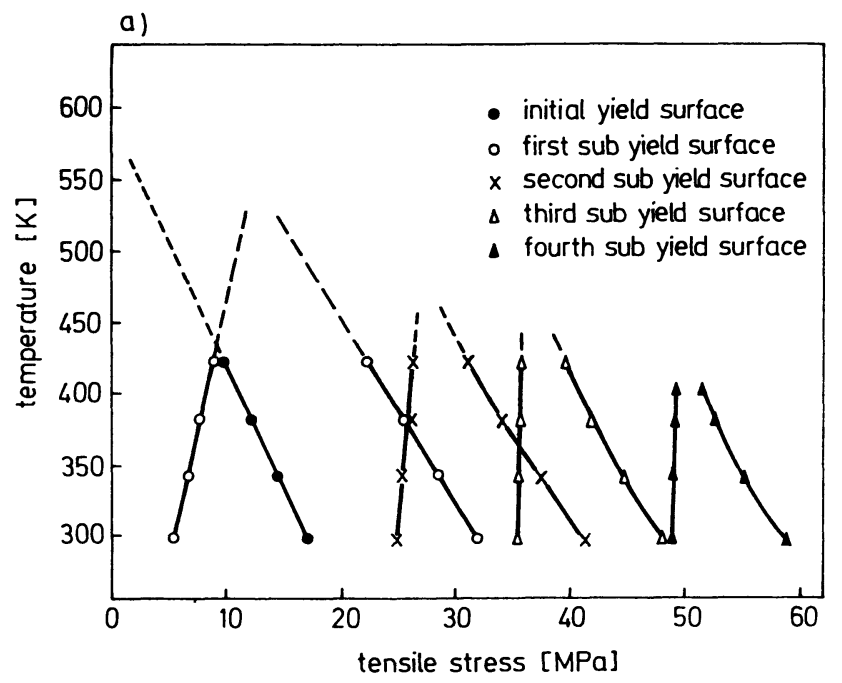

b)

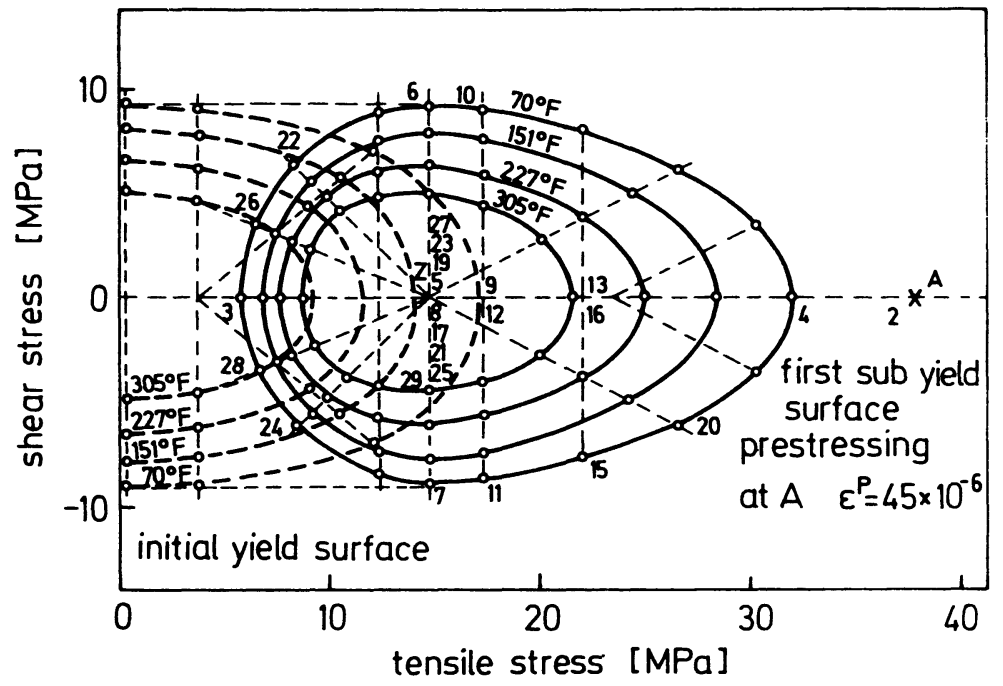

Figure 1 The subsequent yield surfaces at elevated temperature by Phillips and Tang (1972) for initially deformed aluminium specimen: (a) stress-temperature sections of the yield surfaces, (b) and (c) the yield surfaces. 


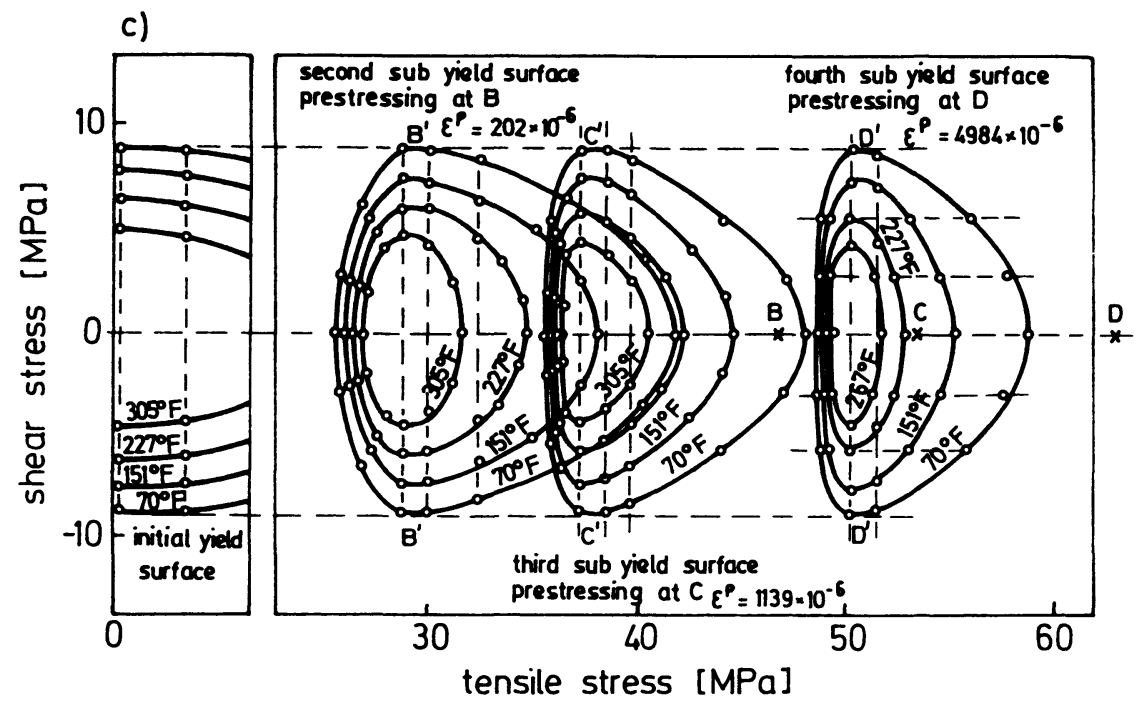

Figure 1 The subsequent yield surfaces at elevated temperature by Phillips and Tang (1972) for initially deformed aluminium specimen: (a) stress-temperature sections of the yield surfaces, (b) and (c) the yield surfaces.

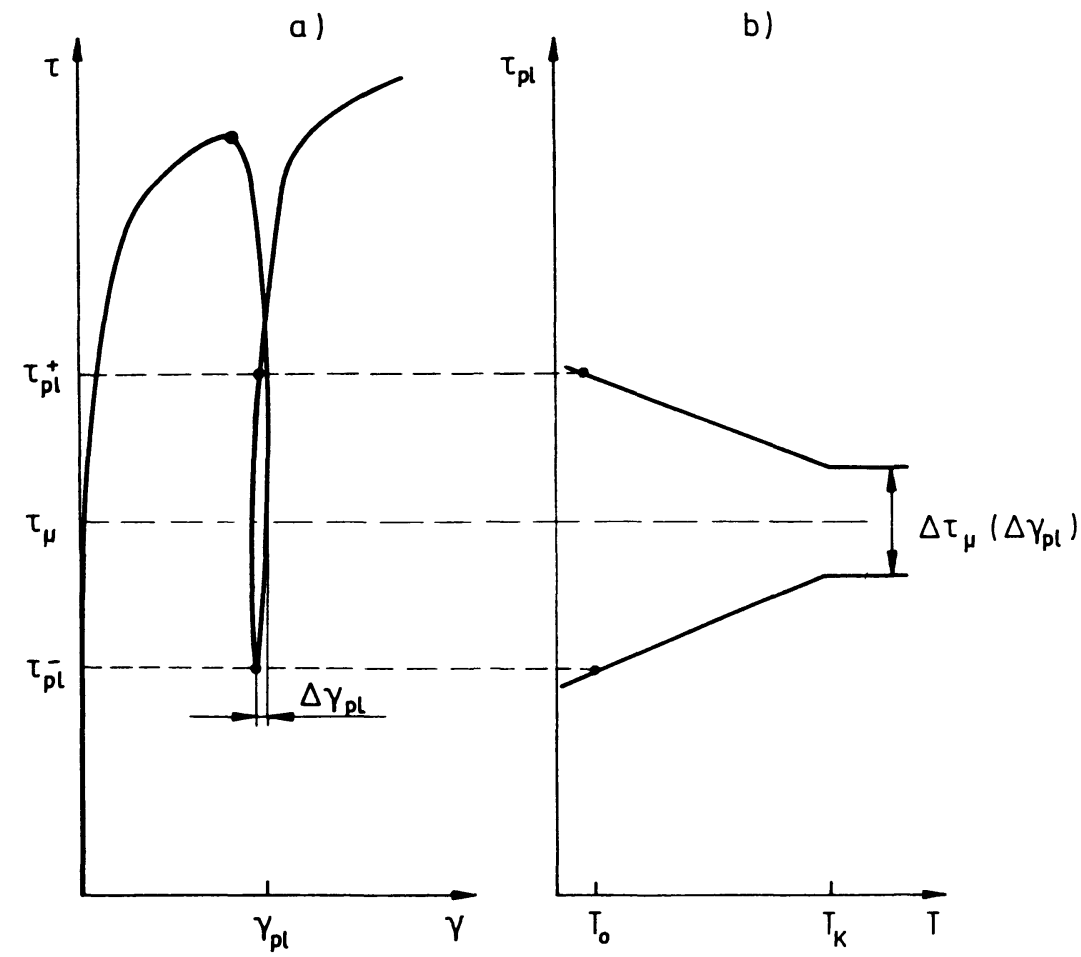

Figure 2 Schematic drawings for plastic flow governed by thermally activated dislocation glides: (a) stress-plastic strain curve for temperature $T_{0}$, (b) dependence of the flow stress $\tau_{\mathrm{pl}}^{ \pm}$corresponding to small strain offsets on the temperature. 


\section{AVERAGING SCHEMES}

Let us assume that the macro-strain of the polycrystal is mainly due to micro-slips in its grains. We limit our considerations to the infinitesimal strain approach for single-phase polycrystal.

The overall strain $\varepsilon$ can be defined as a result of averaging the micro-strains $\hat{\boldsymbol{\varepsilon}}$ over the representative volume region $V_{0}$ of the polycrsytal,

$$
\varepsilon=\frac{1}{\left|V_{0}\right|} \int_{V_{0}} \hat{\varepsilon}(\mathbf{x}) d V
$$

where $\left|V_{0}\right|=\int_{V_{0}} d V$, and

$$
\hat{\mathbf{\varepsilon}}(\mathbf{x})=\sum_{i=1}^{N} \hat{\gamma}(\mathbf{x}) \mathbf{n}^{i}\left(\phi_{\mathrm{cr}}(\mathbf{x})\right)
$$

In the last equation $N$ denotes the number of all crystallographic slip systems of the crystal occupying the point $\mathbf{x}, n^{i}$ determines the tensor of $i$-slip system orientation dependent on the crystal orientation $\phi_{c r}$. The crystal orientation can be described by angular coordinates on the constant curvature Riemannian space of all orientations of the crystal. Then, the orientation is described by contravariant angular coordinates $\left(\phi_{\mathrm{cr}}^{1}, \phi_{\mathrm{cr}}^{2}, \phi_{\mathrm{cr}}^{2}\right)$ which may be identified respectively with the Euler angles $\beta, \eta, \phi$ shown in Figure 3.

Usually, Euler angles determine the crystal orientation with respect to crystal axes. In our case we will use a slightly different relation, let $\mathbf{m} \equiv\langle 111\rangle$ and $\mathbf{s} \equiv\langle 1 \overline{1} 0\rangle$ then the region of all possible orientations of the f.c.c. crystal can be

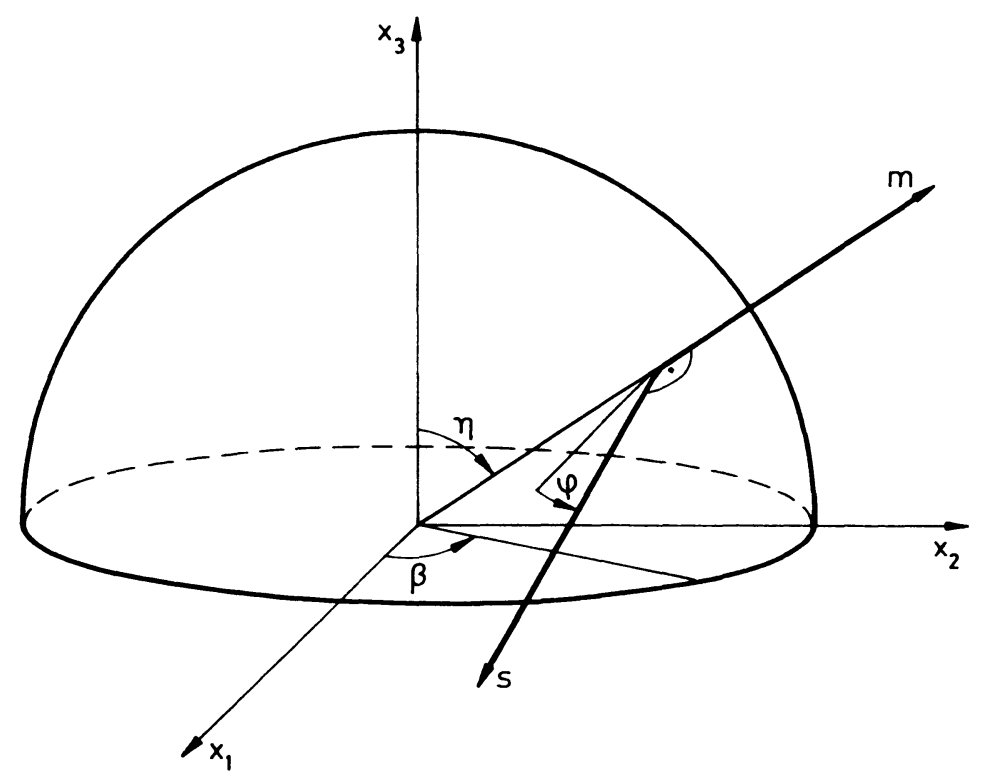

Figure 3 The Euler angles $\beta, \eta, \phi$. 
expressed by

$$
\Omega_{\mathrm{cr}}=\left\{\begin{array}{l}
0 \leq \phi_{\mathrm{cr}}^{1}<\frac{1}{2} \pi \\
0 \leq \phi_{\mathrm{cr}}^{2}<\frac{1}{2} \pi \\
0 \leq \phi_{\mathrm{cr}}^{3}<\frac{1}{3} \pi
\end{array}\right\}+\left\{\begin{array}{l}
0 \leq \phi_{\mathrm{cr}}^{1}<\frac{1}{2} \pi \\
\pi \leq \phi_{\mathrm{cr}}^{2}<\frac{3}{2} \pi \\
0 \leq \phi_{\mathrm{cr}}^{3}<\frac{1}{3} \pi
\end{array}\right\}
$$

Considering the rotation of crystal as a geodesic line in Riemannian space it can be shown that the angle between two different orientations of the crystal is simply the Riemannian distance in the orientation space see Dluzewski (1991).

In (5) we take into account not only active but all crystallographic slip systems. Therefore this equation is independent of the number of active slip systems, however we can expect that at the point $x$ most of the components in (5) are equal zero and correspond to non-active slip systems. For each orientation $\phi_{c r}$ we may determine the strain average

$$
\varepsilon_{\mathrm{cr}}\left(\phi_{\mathrm{cr}}\right)=\lim _{\Delta \phi_{\mathrm{cr}} \rightarrow 0} \frac{1}{\left|V_{\mathrm{cr}}\right|} \int_{V_{\mathrm{cr}}\left(\phi_{\mathrm{cr}} \pm \Delta \phi_{\mathrm{cr}}\right)} \hat{\boldsymbol{\varepsilon}}\left(\mathbf{x}^{\prime}\right) d V^{\prime}
$$

where $V_{\text {cr }}$ denotes the part of the polycrystal region which is occupied by similarly oriented grains, with accuracy $\pm \Delta \phi_{\mathrm{cr}}$, what can be also expressed by

$$
\left\{\mathbf{x} \in V_{\mathrm{cr}}\left(\phi_{\mathrm{cr}} \pm \Delta \phi_{\mathrm{cr}}\right): \bigwedge_{1 \leq i \leq 3}\left(\phi_{\mathrm{cr}}^{i}-\Delta \phi_{\mathrm{cr}}^{i}\right) \leq \phi_{\mathrm{cr}}^{i}(\mathbf{x})<\left(\phi_{\mathrm{cr}}^{i}+\Delta \phi_{\mathrm{cr}}^{i}\right)\right\}
$$

Using (5) and (7) we obtain

$$
\boldsymbol{\varepsilon}_{\mathrm{cr}}\left(\boldsymbol{\phi}_{\mathrm{cr}}\right)=\sum_{i=1}^{N} \gamma_{\mathrm{cr}}^{i}\left(\phi_{\mathrm{cr}}\right) \mathbf{n}^{i}\left(\phi_{\mathrm{cr}}\right)
$$

where

$$
\gamma_{\mathrm{cr}}^{i}\left(\phi_{\mathrm{cr}}\right)=\lim _{\Delta \phi_{\mathrm{cr}} \rightarrow 0} \frac{1}{\left|V_{\mathrm{cr}}\right|} \int_{V_{\mathrm{cr}}\left(\phi_{\mathrm{cr}} \pm \Delta \phi_{\mathrm{cr}}\right)} \hat{\gamma}^{i}\left(\mathbf{x}^{\prime}\right) d V^{\prime}
$$

On the base of (7) the equation (4) may be rewritten as

$$
\varepsilon=\frac{1}{\left|V_{0}\right|} \int_{V_{0}} \varepsilon_{\mathrm{cr}}\left(\phi_{\mathrm{cr}}(\mathbf{x})\right) d V
$$

The volume fraction of all equally oriented crystals is described by the crystal orientation distribution function $F$, see Bunge (1969). This function is defined here as a normalized function

$$
F=\frac{\left|\Omega_{\mathrm{cr}}\right|}{\left|V_{\mathrm{o}}\right|} \frac{d V}{d \Omega},
$$

where $\left|\Omega_{\mathrm{cr}}\right|=\int_{\Omega_{\mathrm{cr}}} d \Omega$. The expression $d V / d \Omega$ denotes the Jacobian determinant of the transition from the integration over the spatial coordinates $\left(x^{1}, x^{2}, x^{3}\right)$ to the integration over angular coordinates and can be understood as

$$
\frac{d V}{d \Omega}=\lim _{\Delta \phi_{\mathrm{cr}} \rightarrow 0} \frac{V_{\mathrm{cr}}\left(\phi_{\mathrm{cr}}+\Delta \phi_{\mathrm{cr}}\right)}{\sqrt{a_{\mathrm{cr}}} \Delta \phi_{\mathrm{cr}}^{1} \Delta \phi_{\mathrm{cr}}^{2} \Delta \phi_{\mathrm{cr}}^{3}},
$$


where $\sqrt{a_{\mathrm{cr}}}$ denotes the determinant of the angular metric tensor in the space of crystal orientations. The function $F$ allows a transition from the integration over the volume region of material in (11) to the integration over crystal orientations, where

$$
\varepsilon=\frac{1}{\left|\Omega_{\mathrm{cr}}\right|} \int_{\Omega_{\mathrm{cr}}} \varepsilon_{\mathrm{cr}}\left(\phi_{\mathrm{cr}}\right) F\left(\phi_{\mathrm{cr}}\right) d \Omega_{\mathrm{cr}}
$$

According to Riemannian geometry $d \Omega_{\mathrm{cr}}=\sqrt{a_{\mathrm{cr}}} d \phi_{\mathrm{cr}}^{1} d \phi_{\mathrm{cr}}^{2} d \phi_{\mathrm{cr}}^{3}$. In the case of Euler angles used as contravariant angular coordinates the expression $\sqrt{a_{\mathrm{cr}}}$ is equal to $\left|\sin \phi_{\mathrm{cr}}^{2}\right|$.

On the base of (14) and (9) we can also obtain the following averaging scheme

$$
\varepsilon=\frac{1}{\left|\Omega_{s}\right|} \int_{\Omega_{s}} \gamma\left(\phi_{s}\right) \mathbf{n}\left(\phi_{s}\right) d \Omega_{s} .
$$

where the strain $\gamma$ is induced by all equally oriented slips in the polycrystal, see Appendix A. $\phi_{s}$ and $\Omega_{s}$ denote the slip orientation and the region of all orientations of slip system, respectively. Using Euler angles, Figure 3, where the unit vector $\mathbf{m}$ is perpendicular to the slip plane and $\mathbf{s}$ is parallel to the slip direction this region can be specified as

$$
\Omega_{s}=\left\{\begin{array}{l}
0 \leq \phi_{s}^{1}<2 \pi \\
0 \leq \phi_{s}^{2}<\frac{1}{2} \pi \\
0 \leq \phi_{s}^{3}<\pi
\end{array}\right.
$$

On the base of the transition from (14) to (15), see Appendix A, we obtain the following relationship for aluminium polycrystal

$$
\gamma\left(\phi_{s}\right)=\frac{\left|\Omega_{s}\right|}{\left|\Omega_{\mathrm{cr}}\right|}\left[\gamma_{\mathrm{cr}}^{A}\left(\phi_{s}\right) F^{A}\left(\phi_{s}\right)+\gamma_{\mathrm{cr}}^{B}\left(\phi_{s}\right) F^{B}\left(\phi_{s}\right)\right]
$$

The indices $A$ and $B$ denote two different crystal orientations corresponding to the fixed orientation of slips, see Figure 4. A similar averaging scheme can be applied to stress distribution. Then the strain average $\gamma$ has a conjugate shear stress.

$$
\tau\left(\phi_{s}\right)=\frac{\left|\Omega_{s}\right|}{\left|\Omega_{\mathrm{cr}}\right|}\left[\tau_{\mathrm{cr}}^{A}\left(\phi_{s}\right) F^{A}\left(\phi_{s}\right)+\gamma_{\mathrm{cr}}^{B}\left(\phi_{s}\right) F^{B}\left(\phi_{s}\right)\right]
$$

where

$$
\tau_{\mathrm{cr}}^{A}\left(\phi_{s}\right)=\lim _{\Delta \phi_{s} \rightarrow 0} \frac{1}{\left|V_{\mathrm{cr}}^{A}\right|} \int_{V_{\mathrm{cr}}^{A}\left(\phi_{s}^{ \pm} \Delta \phi_{s}\right)} \hat{\sigma}\left(x^{\prime}\right) d V^{\prime}
$$

The analogous relation is obtained for the crystal orientation denoted by $B$. For many models, see e.g. Hill (1972), it can be shown that for macroscopically uniform boundary conditions on the volume region $V_{\mathrm{cr}}=V_{\mathrm{cr}}^{A}+V_{\mathrm{cr}}^{\mathrm{B}}$ the principle of virtual work gives

$$
\varphi_{s} \tau\left(\phi_{s}\right) \gamma\left(\phi_{s}\right)=\lim _{\Delta \phi_{s} \rightarrow 0} \frac{1}{\left|V_{\mathrm{cr}}\right|} \int_{V_{\mathrm{cr}}\left(\phi_{s} \pm \Delta \phi_{s}\right)} \hat{\imath}(\mathbf{x}) \hat{\gamma}(\mathbf{x}) d V
$$




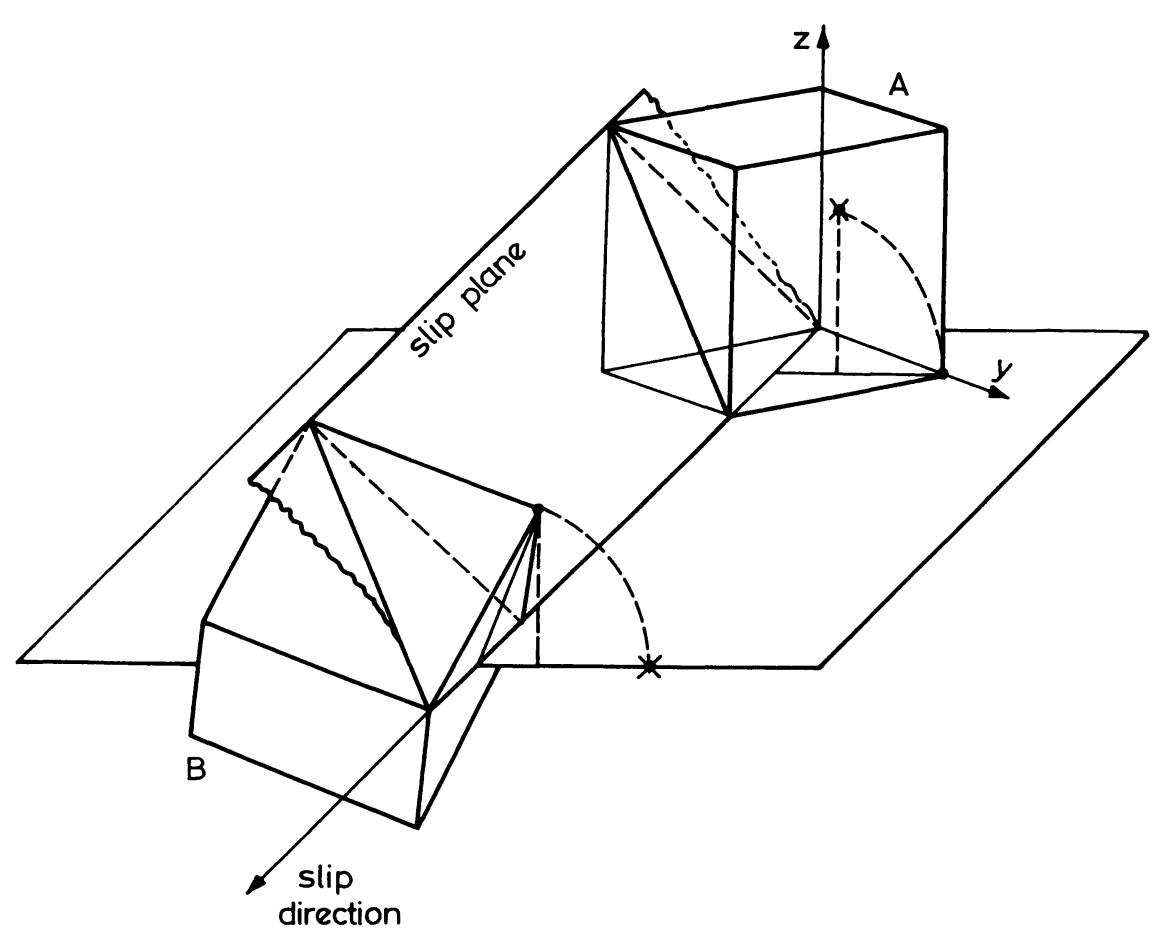

Figure 4 Two different f.c.c. crystal orientations containing the same orientation of the easy glide system.

The results discussed in this section concern many constitutive models applied. Thus, the averaging schemes (4), (14) and (15) we may use to divide constitutive models based on the concept of slip into three groups. The first of them, more general, concerns a modelling of polycrystalline aggregate as a boundary value problem for arbitrary arrangement of grains. Some prototype of such approach has been developed e.g. by Lin and Ito (1966). The second very important group makes the models based indirectly on the scheme (14). According to our results we can say that these models describe only the strain average for all equally oriented grains. The third group discussed below is composed of constitutive models based on the scheme (15).

\section{SLIP THEORY}

In slip theory the stress-strain constitutive relations between the conjugate averages $\gamma$ and $\tau$ or their derivatives are postulated. Slip theory has been used by Batdorf and Budiansky (1949), Como and D'Agostino (1969), Pan and Rice (1983) Dłuzewski (1984) among many others. Usually, the averaging scheme (15) has been assumed a priori. Whereas here, the presented transition from (4) to (15) gives the precise relationship between the micro-strains $\hat{\gamma}(\mathbf{x})$, crystal strain average $\gamma_{c r}$ and the slip strain average $\gamma$. The result of the micro-macro 
transition depends on the crystal symmetry as well as on the crystal orientation distribution.

It is worth to emphasis that in the case of axial loading, slip theory gives the following, very simple relationship for axial strain

$$
\varepsilon=\frac{1}{2 \pi^{2}} \int_{0}^{0.5} \gamma \mu f d \mu,
$$

where $\mu$ is the Schmid factor, $f$ denotes the orientation distribution function of slip systems. The function $f(\mu)$ for untextured polycrystals has been calculated in Appendix C.

Recently, many theories based on the concept of slip are used to predict the deformation behaviour of polycrystal. In generally used models based on the averaging scheme (14) the main attention is often focused on homogeneous deformation of the grains. In slip theory the constitutive equations are postulated for slip systems. Thus, the residual stresses counteracting the slip process will not be modelled here as an effect of interaction of the homogenous grain with its matrix, but it will be simulated as a result of interaction of the relatively large, strongly local slips with approximately rigid walls. In other words we will assume that the elastic accommodation of walls is negligibly small in relation to the strain resolved within slip bands.

\subsection{Constitutive Model}

In our computer simulations the model in which the flow of polycrystalline aluminium was described by the equations (1) and (15) has been applied. It is assumed that below a certain threshold stress $\tau_{\mu 0}$ due to grain boundaries and other obstacles, the evolution of the residual stress $\tau_{\mu}$ is governed by elastic interactions between mobile dislocations and obstacles according to

$$
\dot{\boldsymbol{\tau}}_{\mu}=h \cdot \dot{\gamma}, \text { for }\left|\tau_{\mu}\right| \leq \boldsymbol{\tau}_{\mu 0} .
$$

if the residual stress reached the threshold stress then further deformation is associated with the increase of the dislocation density $\rho$ for this system according to the well known relation

$$
\dot{\rho}=c_{m}|\dot{\gamma}|, \text { for }\left|\tau_{\mu}\right|=\tau_{\mu 0} .
$$

The values of the factor $c_{m}$ for various mono- and polycrystals have been measured by many investigators, see Table 6.1 in Gilman (1969).

Many authors discuss residual stresses as an effect of interactions of glide dislocations with grain boundaries, coplanar dislocations, and with forest dislocations. Here we assume that the increase of the threshold stress is controlled by the change of dislocation densities, where

$$
\tau_{\mu 0}=\tau_{0}+h_{1}(\rho)^{1 / 2}+h_{2}\left(\rho_{\text {tot }}\right)^{1 / 2} .
$$

The second component of the sum predicts the self-hardening effect, while the third is an attempt to estimate the latent-hardening effect. According to the averaging scheme presented above the total dislocation density $\rho_{\text {tot }}$ in the 
Table 1 Material constants assumed for aluminium polycrystal

\begin{tabular}{|c|c|c|}
\hline Symbol & Value & Constant, (Eq.) \\
\hline $\boldsymbol{U}$ & $0.375 \mathrm{eV}$ & Activation energy, (1) \\
\hline $\boldsymbol{v}$ & $5.0 * 10^{-9} \mathrm{~cm}^{3}$ & Activation volume, (1) \\
\hline$c_{m}$ & $1 * 10^{-11} \mathrm{~cm}^{-2}$ & $\begin{array}{l}\text { Dislocation multiplication } \\
\text { coefficient, }(23)\end{array}$ \\
\hline$b \cdot v_{0}$ & $3.2 * 10^{-11} \mathrm{~cm}^{2} \mathrm{~s}^{-1}$ & $\begin{array}{l}\text { Burgers vector/ } \\
\text { velocity coefficient, (1) }\end{array}$ \\
\hline$f_{0}$ & 1 & Mobile dislocation \\
\hline$\alpha$ & $1 * 10^{-11} \mathrm{~cm}^{2}$ & factor, (26) \\
\hline$\beta$ & $3 * 10^{-9} \mathrm{~cm}^{2}$ & \\
\hline$h$ & $2.4 * 10^{4} \mathrm{MPa}$ & Hardening \\
\hline$\tau_{0}$ & $14.5 \mathrm{MPa}$ & coefficients, (22), (24) \\
\hline$h_{1}$ & $7.9 * 10^{4} \mathrm{~cm}$ & \\
\hline$h_{2}$ & $2.7 * 10^{5} \mathrm{~cm}$ & \\
\hline
\end{tabular}

polycrystal is determined by

$$
\rho_{\mathrm{tot}}=\frac{1}{\left|\Omega_{s}\right|} \int_{\Omega_{s}} \rho d \Omega_{s} .
$$

It is assumed here after Gilman (1969), that the mobile dislocations $\rho_{m}$ compose a fraction of all dislocations for the slip system,

$$
\rho_{m}=f_{m} \rho,
$$

where $f_{m}$ denotes the fraction factor. In our approach the factor is predicted by the following rule $f_{m}=f_{0} \cdot \exp (-\alpha \rho) \cdot \exp \left(-\beta \rho_{\text {tot }}\right)$. According to the averaging scheme derived in the previous section the total density of mobile dislocations in the polycrystal is determined by

$$
\rho_{m_{\mathrm{tot}}}=\frac{1}{\left|\Omega_{s}\right|} \int_{\Omega_{s}} \rho_{m} d \Omega_{s}
$$

\subsection{Computer procedure}

The values of the constants assumed for the calculations are presented in Table 1. A rheological character of the thermal activation of slips (1) forced us to simulate the whole flow process observed by investigators. Therefore the process has been numerically simulated step by step in time according to the data on the experimental procedure.

The numerical calculations were carried out for various discretizations of the slip system space $\Omega_{s}$. The most general discretization was composed with the slip systems variously oriented in space, Dluzewski (1984). A simple discretization of the slip space obtained for two-dimensional loadings $\sigma-\tau$ is shown in Appendix B.

\section{RESULTS}

The investigation of the yield surfaces has been numerically simulated taking into account the rate and direction of loading as well as the criteria of yielding similar 
to those which have been used by Phillips and Tang (1972) in their experimental test. In our computer simulations the injection of the yield surface started in the direction of preloading and was continued subsequently in reversed directions until the orthogonal direction to the preloading has been reached.

Figure 5 shows the results of computer simulation for aluminium observed after subsequent axial preloadings shown in Figure 1. With respect to the difficulties in a precise presentation of all the yield points for subsequent yield surfaces, these points have been omitted in Figure 5b. An example of the yield point distributions obtained for one yield surface will be shown in Figure 9. Figure 6a shows the surfaces obtained experimentally for the combined loading path starting with the torsion to point A. Figure $6 \mathrm{~b}$ shows the surfaces obtained
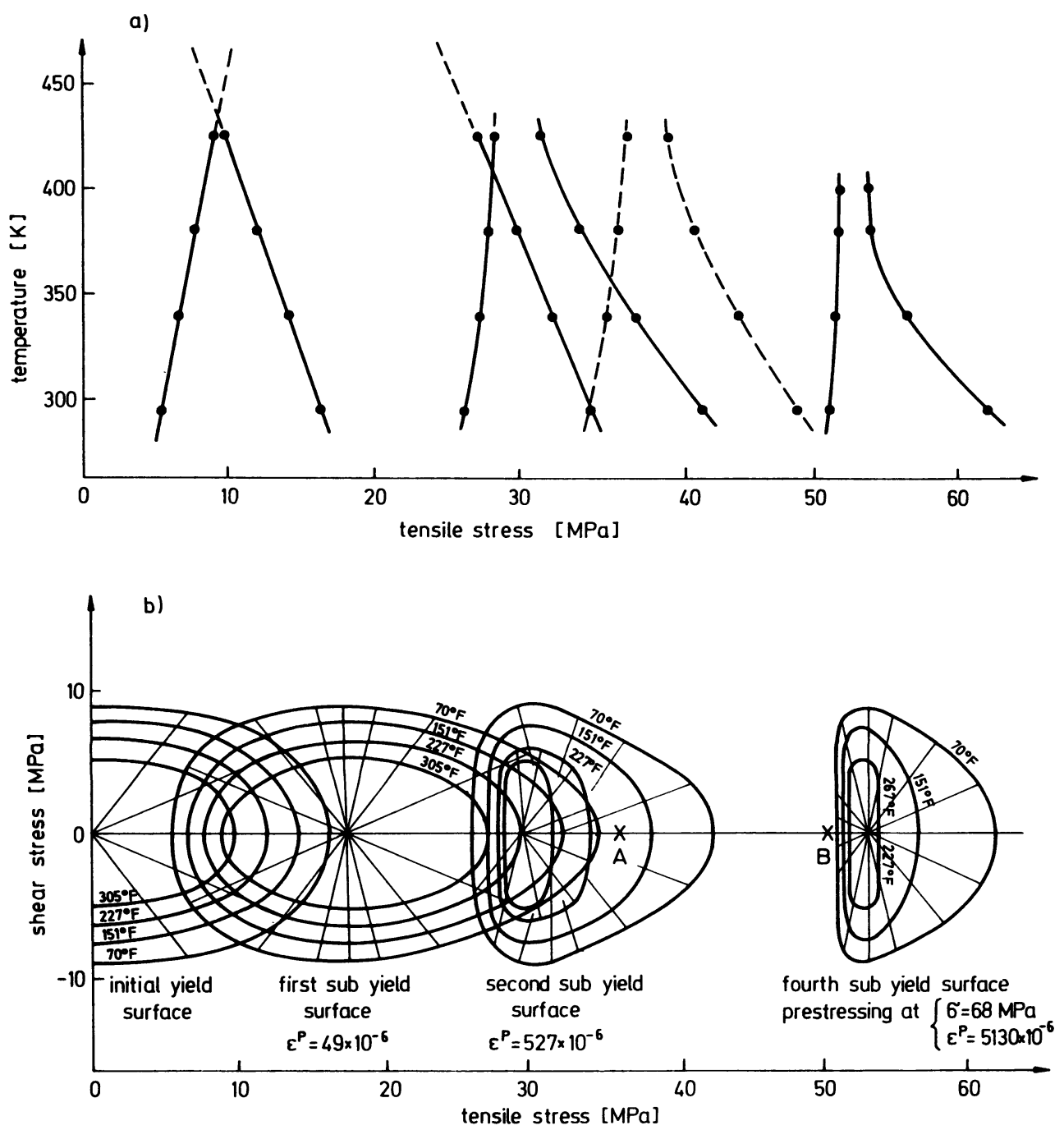

Figure 5 Numerically obtained subsequent yield surfaces: (a) stress-temperature sections of the yield surfaces, (b) the yield surfaces. 
a)
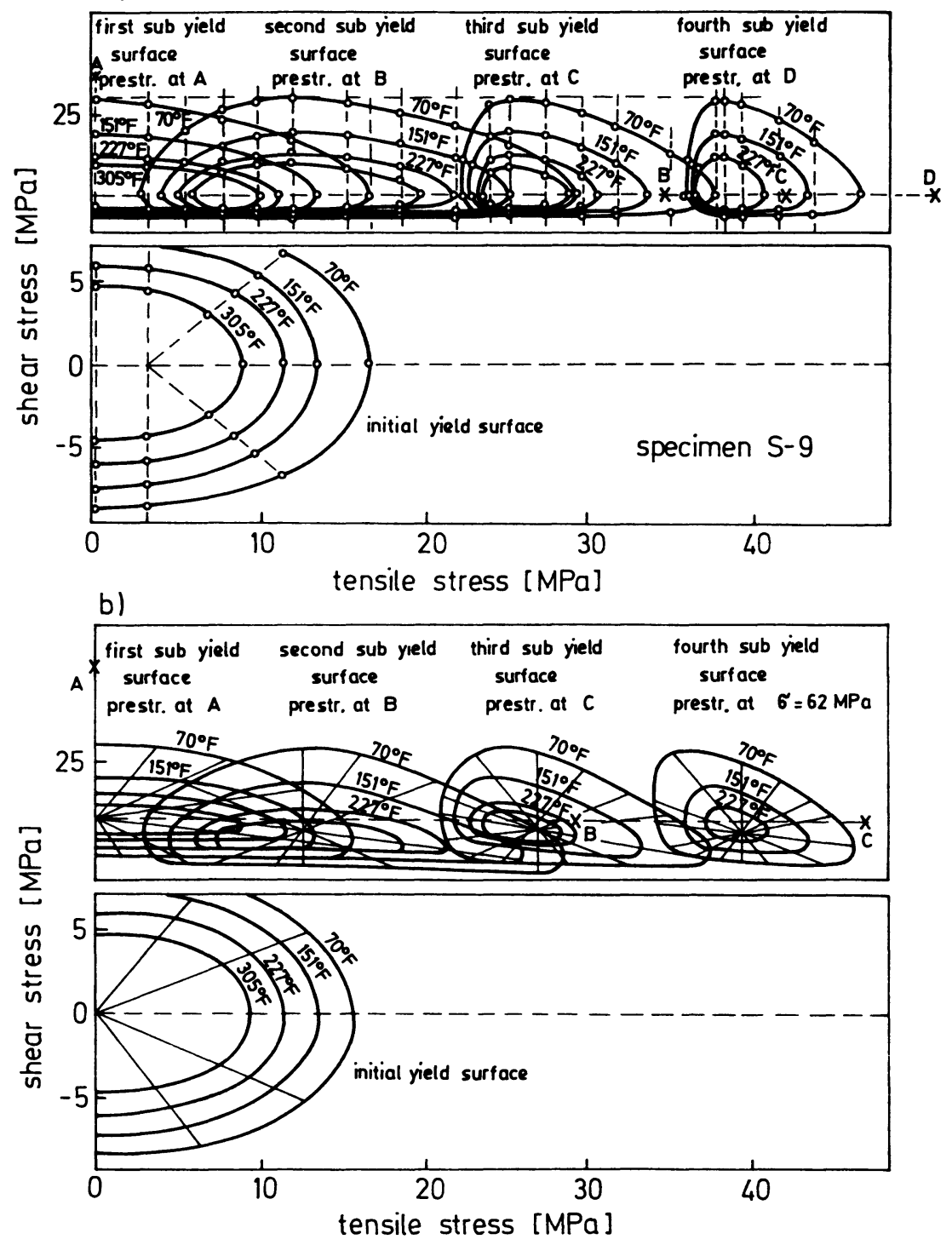

Figure 6 Comparison of the subsequent yield surfaces: (a) from experimental procedure Phillips and Tang (1972), (b) from numerical investigation of the behaviour of the constitutive model based on the slip theory.

as a result of the response of the numerical model for the analogous preloading path. Figure 7 shows the comparison of yield surfaces for another preloading path.

The distance between reversely directed flow stresses on the yield surface depends on the mobile dislocation density. To illustrate the dislocation density 

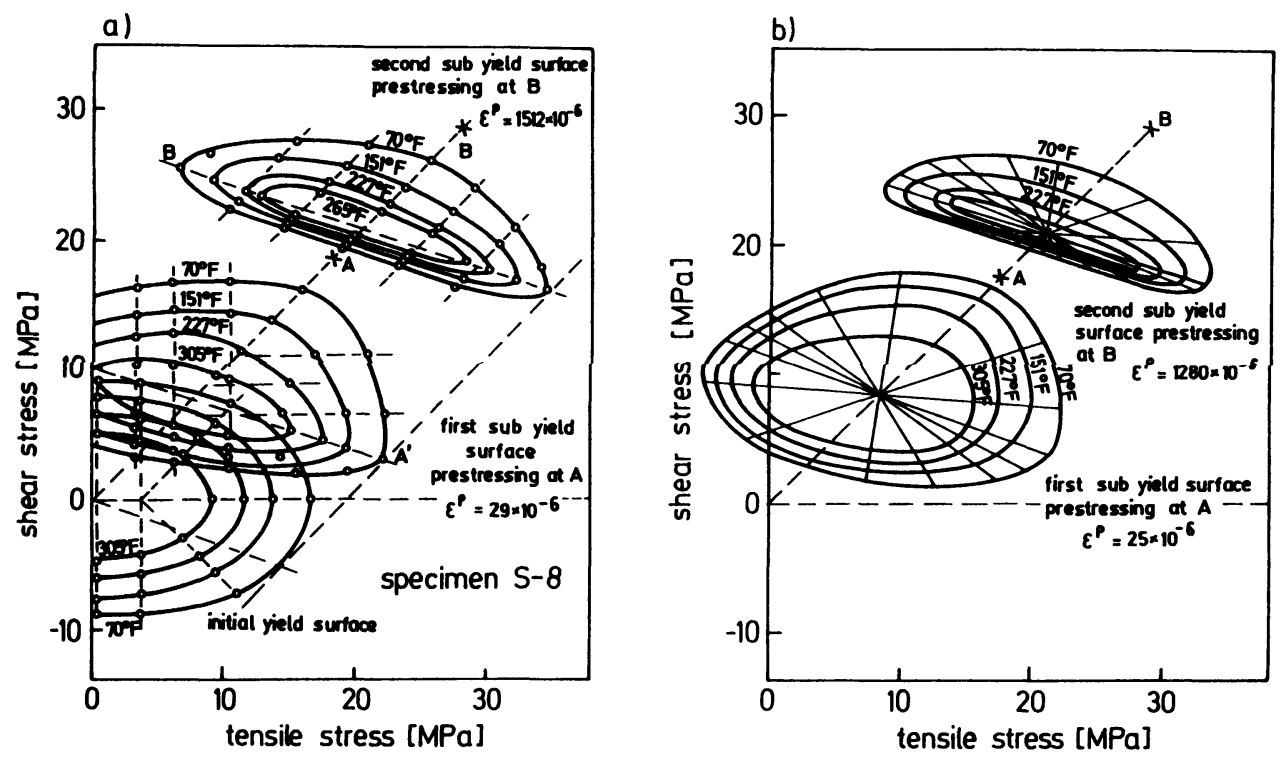

Figure 7 Comparison of the subsequent yield surfaces: (a) from experimental procedures Phillips and Tang (1972), (b) from numerical investigation of the behaviour of the constitutive model based on the slip theory.

evolution obtained in our calculations the process of pure shearing has been computed for the rate of shear $1 * 10^{-2} \mathrm{MPa} / \mathrm{s}$, Figure 8 . In our case the dislocation density for one orientation of slips is described by $\rho$ and $\rho_{m}$ while the densities referred to the volume $V_{\text {cr }}$ are determined by

$$
\rho_{\mathrm{cr}}=\frac{\left|\Omega_{\mathrm{cr}}\right|}{2\left|\Omega_{s}\right|} \rho
$$

and

$$
\rho_{m_{\mathrm{cr}}}=\frac{\left|\Omega_{\mathrm{cr}}\right|}{2\left|\Omega_{s}\right|} \rho_{m}
$$

Similarly,

$$
\gamma_{\mathrm{cr}}=\frac{\left|\Omega_{\mathrm{cr}}\right|}{\left|\Omega_{s}\right|} \gamma
$$

where $\gamma_{\text {cr }}$ denotes the shear strain averaged over the total volume of all crystals $A$ and $B$ in which such oriented easy glide system exists, see Appendix A.

\section{CONCLUDING REMARKS}

In the present approach the yield surface is obtained by simulation of the deformation process corresponding to the applied definition of yielding. There- 

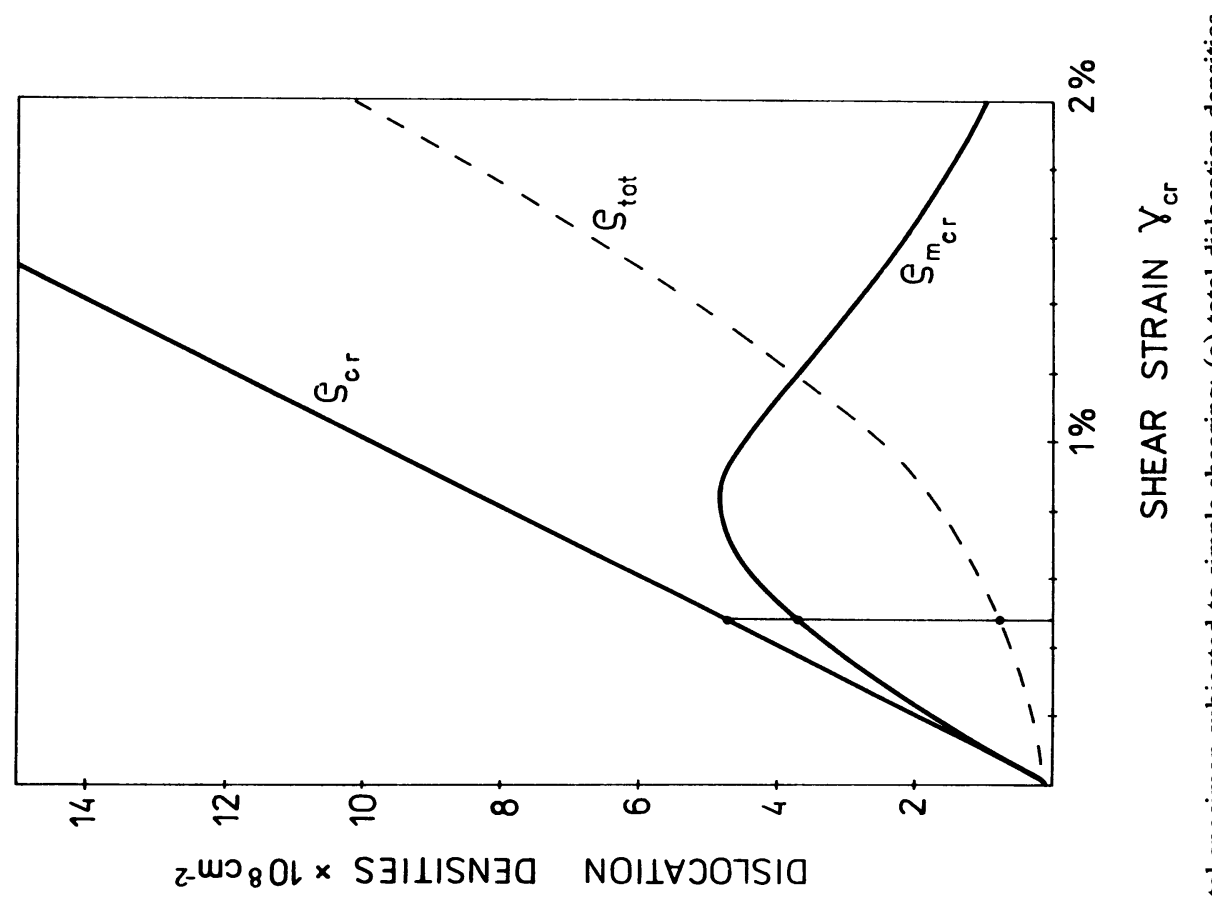

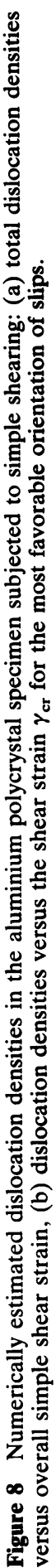

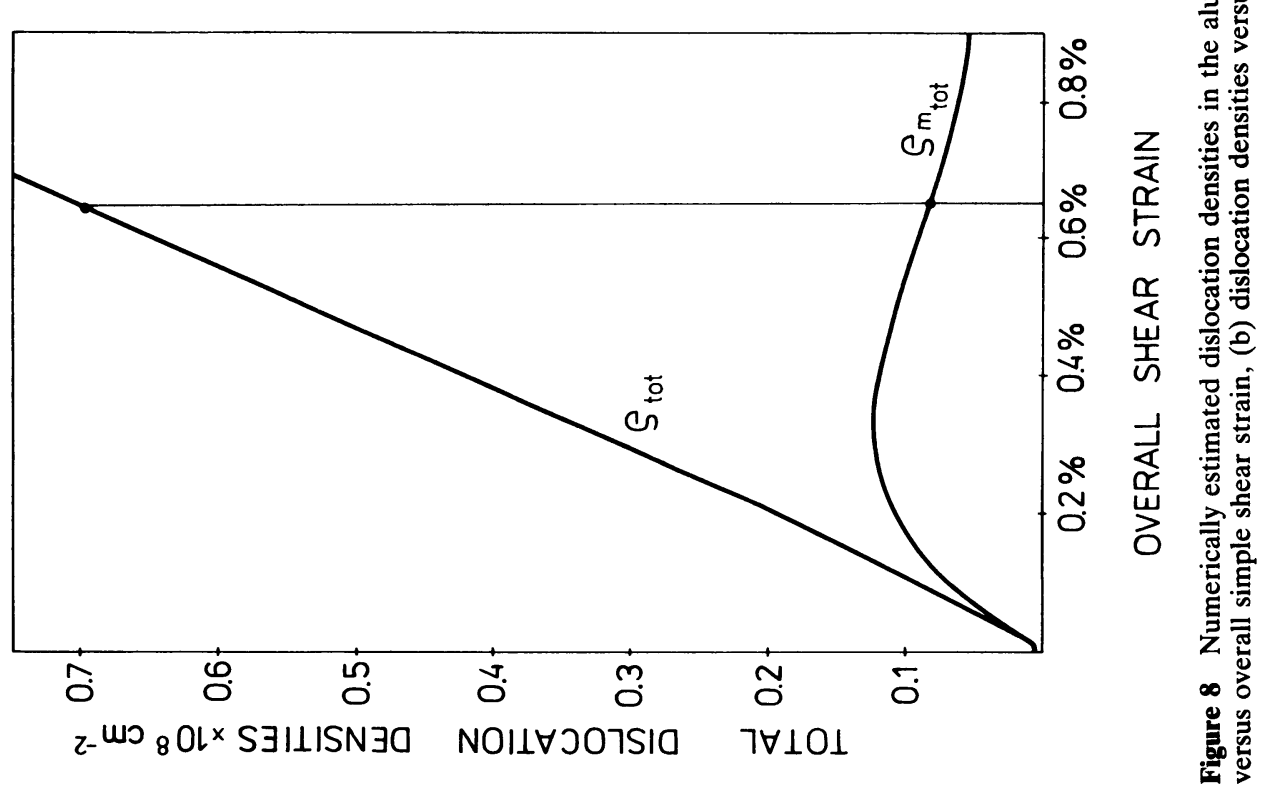




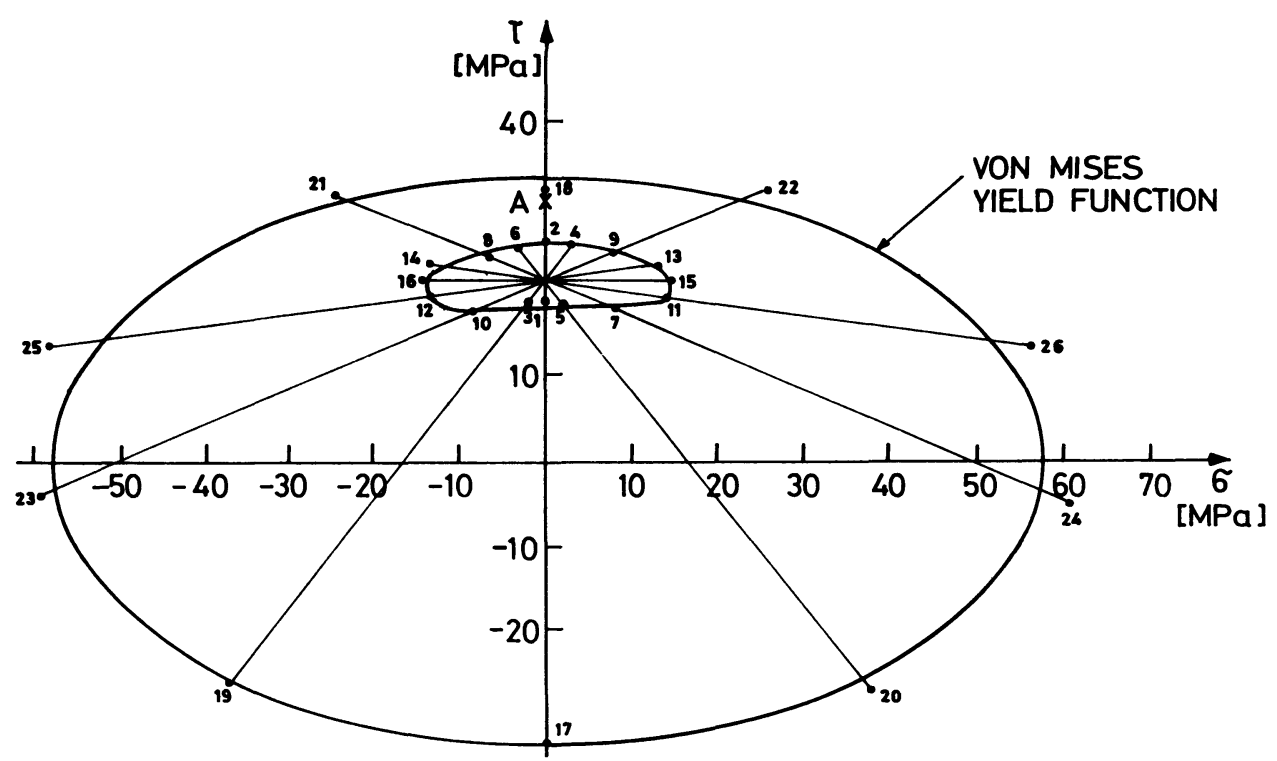

Figure 9 Numerically obtained yield surfaces for aluminium. After pre-shear-straining of $0.3 \%$ at $70^{\circ} \mathrm{F}$ to point $\mathrm{A}$ and partial unloading the yield surface was tested for the offset $5 * 10^{-6}$. Next, using $0.1 \%$ offset the points 17 to 26 were obtained.

fore, the yield stress is not an inherent part of the used constitutive model, but depends on the used definition of yielding. For example, assuming two different strain offsets $5 * 10^{-6}$ and $0.1 \%$, two different yield surfaces are obtained in one deformation process, see Figure 9. These results are in general agreement with experimental data, see Szczepinski (1963), Stout et al. (1985).

To determine the yield stress investigators are forced to induce in the material a certain plastic strain offset, usually $0.2 \%$. A significant reduction of the offset below $1 * 10^{-5}$, made by Phillips and Tang (1972), gives also a significant reduction of the observed athermal stresses. This experimental evidence suggests that the experimentally obtained athermal stress is due to residual stresses induced by the applied plastic strain offset. The relatively small polycrystal strain, e.g. $0.2 \%$ induces large internal stresses, cf. Figure $1 \mathrm{a}$, and consequently leads to a deviation from the linearity of the yield stress-temperature diagram.

\section{APPENDIX A}

Using (9) the Eq. (14) can be rewritten as

$$
\varepsilon=\frac{1}{\left|\Omega_{\mathrm{cr}}\right|} \int_{\Omega_{\mathrm{cr}} i=1}^{N} \sum_{\mathrm{cr}}^{i}\left(\phi_{\mathrm{cr}}\right) \mathbf{n}_{\mathrm{cr}}^{i}\left(\phi_{\mathrm{cr}}\right) F\left(\phi_{\mathrm{cr}}\right) d \Omega_{\mathrm{cr}}
$$

The number of all easy glide systems in aluminium crystal is determined here as $N=12$. It is known that for a given crystal orientation $\phi_{c r}$ we can uniquely 
determine the angular coordinates of the slip system i.e.

$$
\boldsymbol{\phi}_{s}=\hat{\boldsymbol{\phi}}_{s}\left(\phi_{\mathrm{cr}}, i\right)
$$

In many cases the inverse relation with respect to $\phi_{\mathrm{cr}}$ does not exist, e.g. in the case of the f.c.c. crystal for each orientation of a slip system we find two mutually different crystal orientations in which such oriented easy glide system exists, see Figure 4. However, in the neighbourhood of $i$-slip system orientation of the crystal one of the following unique relations takes place

$$
\phi_{\mathrm{cr}}=\phi_{\mathrm{cr}}^{A}\left(\phi_{s}, i\right)
$$

or

$$
\phi_{\mathrm{cr}}=\boldsymbol{\phi}_{\mathrm{cr}}^{B}\left(\phi_{s}, i\right)
$$
It can be shown that the jacobian of the transformation $\left|\frac{d \phi_{\mathrm{cr}}}{d \phi_{s}}\right|$ is determined for
(A3) and (A4) as

$$
\left|\frac{d \phi_{\mathrm{cr}}}{d \phi_{s}}\right|=\frac{\sqrt{a_{\mathrm{cr}}}}{\sqrt{a_{s}}}
$$

where $\sqrt{a_{s}}$ and $\sqrt{a_{\mathrm{cr}}}$ denote the roots of the determinant of the angular metric tensors for the orientation spaces of slip and crystal orientations, respectively. In the case of Euler angles used as angular coordinates we obtain $\sqrt{a_{s}}=\left|\sin \phi_{s}^{2}\right|$ and $d \Omega_{s}=\sqrt{a_{s}} d \phi_{s}^{1} d \phi_{s}^{2} d \phi_{s}^{3}$. Analogous relations are satisfied by $\sqrt{a_{\mathrm{cr}}}$ and $d \Omega_{\mathrm{cr}}$. So using the theorem of integration by substitution it follows

$$
\varepsilon=\frac{1}{\left|\Omega_{s}\right|} \int_{\Omega_{s}} \frac{\left|\Omega_{s}\right|}{\left|\Omega_{\mathrm{cr}}\right|}\left[\gamma^{A}\left(\phi_{s}\right) F^{A}\left(\phi_{s}\right)+\gamma^{B}\left(\phi_{s}\right) F^{B}\left(\phi_{s}\right)\right] \mathbf{n}\left(\phi_{s}\right) d \Omega_{s} .
$$

For untextured aluminium we may assume that $F^{A}=F^{B}=1$. Then

$$
\boldsymbol{\varepsilon}=\frac{1}{\left|\Omega_{s}\right|} \int_{\Omega_{s}} \frac{\left|\Omega_{s}\right|}{\left|\Omega_{\mathrm{cr}}\right|} 2 \gamma_{\mathrm{cr}} \mathbf{n} d \Omega
$$

where $\gamma_{\mathrm{cr}}$ denotes the strain average over the total volume occupied by the crystal orientations $A$ and $B$, so

$$
\gamma_{\mathrm{cr}}\left(\boldsymbol{\phi}_{s}\right)=\lim _{\Delta \boldsymbol{\phi}_{s} \rightarrow 0} \frac{1}{\left|V_{\mathrm{cr} \mid}\right|} \int_{V_{\mathrm{cr}}\left(\boldsymbol{\phi}_{s} \pm \Delta \phi_{s}\right)} \hat{\gamma}\left(\mathbf{x}^{\prime}\right) d V^{\prime}=\frac{1}{2}\left(\gamma_{\mathrm{cr}}^{A}+\gamma_{\mathrm{cr}}^{B}\right)
$$

where $V_{\mathrm{cr}}=V_{\mathrm{cr}}^{A}+V_{\mathrm{cr}}^{B}$.

\section{APPENDIX B}

Let us consider the biaxial loading $\sigma_{33}, \sigma_{31}=\sigma_{13}$. Note that to calculate the shear stress $\tau$ for arbitrarily oriented slip system we need two coefficients $n_{\sigma}$ and $n_{\tau}$ where $\tau(\beta, \eta, \phi)=\sigma_{33} \cdot n_{\sigma}(\beta, \eta, \phi)+\sigma_{13} \cdot n_{\tau}(\beta, \eta, \phi)$. On the base of (15) and 
Table B1 The section of slip system space for states of loading $\sigma-\tau$

\begin{tabular}{lllllllrl}
\hline $\begin{array}{l}\text { Slip system } \\
\text { no. }\end{array}$ & $n_{\sigma}$ & \multicolumn{1}{l}{$n_{\tau}$} & $\Delta \Omega$ & $\begin{array}{l}\text { Slip system } \\
\text { no. }\end{array}$ & \multicolumn{1}{l}{$n_{\sigma}$} & \multicolumn{1}{c}{$n_{\tau}$} & $\Delta \Omega$ \\
\hline 1 & & & & & & & \\
2 & 0.000 & 1.000 & 0.0073 & 14 & 0.379 & 0.386 & 0.0435 \\
3 & 0.155 & 0.951 & 0.0076 & 15 & 0.420 & 0.133 & 0.0416 \\
4 & 0.294 & 0.809 & 0.0088 & 16 & 0.420 & -0.133 & 0.0416 \\
5 & 0.404 & 0.588 & 0.0120 & 17 & 0.379 & -0.386 & 0.0435 \\
6 & 0.476 & 0.309 & 0.0217 & 18 & 0.301 & -0.601 & 0.0415 \\
7 & 0.500 & 0.000 & 0.0251 & 19 & 0.193 & -0.757 & 0.0254 \\
8 & 0.476 & -0.309 & 0.0217 & 20 & 0.067 & -0.840 & 0.0233 \\
9 & 0.404 & -0.588 & 0.0120 & 21 & 0.085 & 0.523 & 0.0886 \\
10 & 0.294 & -0.809 & 0.0088 & 22 & 0.223 & 0.323 & 0.0769 \\
11 & 0.155 & -0.951 & 0.0076 & 23 & 0.275 & 0.000 & 0.0570 \\
12 & 0.067 & 0.840 & 0.0233 & 24 & 0.223 & -0.323 & 0.0769 \\
13 & 0.193 & 0.757 & 0.0254 & 25 & 0.085 & -0.523 & 0.0886 \\
& 0.301 & 0.601 & 0.0415 & 26 & 0.000 & 0.000 & 0.1288 \\
\hline
\end{tabular}

(25) we obtain

$$
\left\{\begin{array}{l}
\dot{\varepsilon}_{33} \cong \sum_{i=1}^{N} \dot{\gamma}^{i} n_{\sigma}^{i} \Delta \Omega^{i} \\
\dot{\varepsilon}_{13} \cong \frac{1}{2} \sum_{i=1}^{N} \dot{\gamma}^{i} n_{\tau}^{i} \Delta \Omega^{i} \\
\rho_{\mathrm{tot}} \cong \sum_{i=1}^{N} \rho^{i} \Delta \Omega^{i}
\end{array}\right.
$$

where $\Delta \Omega^{i}=\frac{1}{\left|\Omega_{s}\right|} \int_{\Delta \Omega_{s}^{i}} d \Omega$.

The volume of the slip system space $\Omega_{s}$ has been divided between $N=26$ systems. The whole space was searched with the steps $\Delta \eta, \Delta \beta / \sin (\eta-\Delta \eta / 2)$, $\Delta \phi=2^{\circ}$. Each of the small cells with the dimensions $\Delta \beta, \Delta \eta, \Delta \phi$ was assigned to the nearest system, using as a criterion the minimum of the metric $m=$ $\sqrt{\left(n_{\sigma}^{i}-n_{33}\right)^{2}+2\left(n_{\tau}^{i} / 2-n_{13}\right)^{2}}$. On this way the section of $\Omega_{s}$ into 26 subregions has been obtained, see Table B1.

\section{APPENDIX C}

In the case of uniaxial loading the axial strain $\varepsilon_{33}$ induced by differently oriented slips in the untextured polycrystal is determined by

$$
\varepsilon_{33}=\frac{1}{\left|\Omega_{s}\right|} \int_{\Omega_{s}} \gamma n_{33} d \Omega=\frac{1}{2 \pi^{2}} \int_{\beta=0}^{2 \pi} \int_{\eta=0}^{\pi / 2} \int_{\phi=0}^{\pi} \gamma \mu \sin \eta d \beta d \eta d \phi .
$$

This integrand does not depend on the angle $\beta$, and the Schmid factor is 
determined by $\mu=0.5 \sin 2 \eta \sin \phi$, so for a fixed $\eta$

$$
d \phi=\frac{d \mu}{\sqrt{\left(\frac{\sin 2 \eta}{2}\right)^{2}-\mu^{2}}}
$$

Let us replace the integral variable $\phi$ by $\mu$, then

$$
\varepsilon_{33}=\frac{1}{2 \pi^{2}} \int_{\eta=0}^{\pi / 2} \int_{\phi=0}^{\pi} \gamma \mu 2 \pi \sin \eta d \eta d \phi=\frac{1}{2 \pi^{2}} \int_{\eta=0}^{\pi / 2} \int_{\mu=0}^{(\sin 2 \eta) / 2} \gamma \mu \frac{2 \pi \sin \eta d \eta d \mu}{\sqrt{\left(\frac{\sin 2 \eta}{2}\right)^{2}-\mu^{2}}}
$$

In the last integral, the Schmid factor $\mu$ is independent of the variable $\eta$ as the second independent integral variable. Hence, according to slip theory the shear stress $\tau$ as well as the strain $\gamma$ are also independent of $\eta$. So,

$$
\varepsilon_{33}=\frac{1}{2 \pi^{2}} \int_{\mu=0}^{0.5} \gamma \mu\left[\int_{\eta_{1}=0.5 \arcsin 2 \mu}^{\pi-\eta_{1}} \frac{2 \pi \sin \eta}{\sqrt{\cos ^{2} \eta \sin ^{2} \eta-\mu^{2}}} d \eta\right] d \mu .
$$

Denoting

$$
f(\mu)=\int_{0.5 \arcsin (2 \mu)}^{\pi-0.5 \arcsin (2 \mu)} \frac{2 \pi \sin \eta}{\sqrt{\cos ^{2} \eta \sin ^{2} \eta-\mu^{2}}} d \eta
$$

we obtain (21).

\section{References}

Batdorf, S. B. and Budiansky, B. (1949). A Mathematical Theory of Plasticity Based on the Concept of Slips. NACA Tech. Notes, 1871.

Boas, W. and Ogilvie, G. J. (1954). The plastic deformation of a crystal in a polycrystalline aggregate. Acta metall., 2, 655.

Budiansky, B. and Wu, T. T. (1962). Theoretical prediction of plastic strains of polycrystals. Proc. 4-th Congr. Appl. Mech., 1175.

Bunge, H. J. (1969). Mathematische Methoden der Texturanalyse, Academic Verlag, Berlin.

Como, M. and D'Agostino, S. (1969). Strain hardening plasticity with Bauschinger effect. Meccanica, 4, 146.

Conrad, H. (1961). In Mechanical Behavior of Materials at Elevated Temperatures, J. E. Dorn, ed., McGraw-Hill Book Company, New York.

Dluzewski, P. H. (1984). Slip theory and inelastic deformations; relations between the theory and the experimental results. Arch. Mech., 36, 173-183.

Dłuzewski, P. H. (1987). Some remarks on the integration domain of the slip orientations. Arch. Mech., 39, Brief Notes, 419-422.

Dluzewski, P. H. (1990). Application of slip theory to the prediction of the hardening process induced by inelastic deformations of polycrystals, in Yielding Damage and Failure of Anisotropic Solids, $\mathrm{J}$. P. Boehler, ed. p.p. 221-233, Mechanical Engineering Publications, London.

Dluzewski, P. H. (1991). Crystal orientation spaces and remarks on the modelling of polycrystal anisotropy. J. Mech. Phys. Solids, 39, 651-661.

Gilman, J. J. (1969). Micromechanics of Flow in Solids, McGraw-Hill Book Company, New York.

Hill, R. (1972). On constitutive macro-variables for heterogeneous solids at finite strain. Proc. R. Soc. Lond. A, 326, 131-147.

Hutchinson, J. W. (1970). Elastic-plastic behaviour of polycrystalline metals and composites. Proc. $R$. Soc. Lond. A, 319, 247-272.

Ikegami, K. (1982). Experimental plasticity on the anisotropy of metals, in Mechanical Behavior of Anisotropic Solids, J.-P. Boehler ed., Martinus Nijhoff Publishers, The Hague. 
Kocks, U. F., Argon, A. S. and Ashby, M. F. (1975). Prog. Mat. Sci., 19.

Kiryk, R. and Dluzewski, P. H. (1989). Influence of microstresses on subsequent yield surfaces of polycrystalline materials. Int. J. Engng. Sci., 27, 1589-1592.

Kroner, E. (1961). Zur Plastischen Verformung des Vielkristalls. Acta metall., 9, 155.

Kroner, E. and Teodosiu, C. (1974). Lattice defect approach to plasticity and viscoplasticity, in Problems of Plasticity, A. Sawczuk ed., Noordhoff Int. Publ. Leyden.

Lin, T. H. and Ito, Y. M. (1966). Theoretical plastic stress-strain relationship of a polycrystal and comparison with von Mises and Tresca's plasticity theories. Int. J. Engng. Sci., 4, 543.

Pan, I. and Rice, J. R. (1983). Rate sensitivity of plastic flow and implications for yield surface vertexes. Int. J. Solids Structures, 19, 977.

Phillips, A. and Tang, J. L. (1972). The effect of loading path on the yield surface at elevated temperature. Int. J. Solids Structures, 8, 463.

Stout, M. G., Martin, P. L., Helling, D. E., and Canova, G. R. (1985). Multiaxial yield behavior of 1100 aluminium following various magnitudes of prestrain. Int. J. Plasticity, 1, 163.

Seeger, A. (1954). The temperature dependence of the critical shear stress and of work-hardening of metal crystal. Phil. Mag., 45, 771.

Seeger, A. (1955). The generation of lattice defect by moving dislocation and its application to the temperature dependence of the flow stress of FCC crystals. Phil. Mag., 46, 1194.

Szczepinski, W. (1963). On the effect of plastic deformation on the yield condition. Arch. Mech., 2, 15.

Tokuda, M., Kratochvil, J. and Ohno, N. (1985). Inelastic behaviour of polycrystalline metals under complex loading condition. Int. J. Plasticity, 1, 141.

Weertman, J. (1957). Steady-state creep through dislocation climb. J. Appl. Phys., 28, 1185. 